\title{
A Comparative Assessment of the Surface Roughness of Thermoplastic Denture Base Resins Following Adjustment and Re-Polishing
}

\author{
Shivaughn M. Marchan ${ }^{*}$, Anna Kay Bishop1, William A. J. Smith1, Paul Seerattan², David Hinds ${ }^{3}$ \\ ${ }^{1}$ Unit of Restorative Dentistry, School of Dentistry, Faculty of Medical Sciences, The University of the West Indies, St Augustine, \\ Trinidad and Tobago \\ ${ }^{2}$ School of Dentistry, Faculty of Medical Sciences, the University of the West Indies, St Augustine, Trinidad and Tobago \\ ${ }^{3}$ Department of Physics, Faculty of Science and Technology, the University of the West Indies, St Augustine, Trinidad and Tobago \\ Email: ^Shivaughn.Marchan@sta.uwi.edu
}

How to cite this paper: Marchan, S.M., Bishop, A.K., Smith, W.A.J., Seerattan, P. and Hinds, D. (2017) A Comparative Assessment of the Surface Roughness of Thermoplastic Denture Base Resins Following Adjustment and Re-Polishing. Open Journal of Stomatology, 7, 250-263.

https://doi.org/10.4236/ojst.2017.74019

Received: January 3, 2017

Accepted: April 27, 2017

Published: April 30, 2017

Copyright $\odot 2017$ by authors and Scientific Research Publishing Inc. This work is licensed under the Creative Commons Attribution International License (CC BY 4.0).

http://creativecommons.org/licenses/by/4.0/

cC) (i) Open Access

\begin{abstract}
Purpose: This study assessed the roughness of two injection-molded, thermoplastic materials used for denture bases compared with a polyamide material and compression molded Polymethylmethacrylate (PMMA) after the adjustment and re-polishing with either a laboratory protocol or a chair side protocol. Methods: Forty specimens, each of PMMA, Valplast, DuraFlex, Dura Cetal were fabricated and finished according to individual manufactures' instructions. These materials were adjusted with tungsten carbide (TC) burs to mimic gross adjustments, and then re-polished either on a lathe or bonded silicon carbide (B-SC). Following instrumentation, the specimens were assessed using contact profilometry and scanning electron microscopy. Two-factor ANOVA was used to determine significant differences in mean surface roughness $\left(R_{a}\right.$ and $\left.R_{\max }\right)$, with included factors being material type and re-polishing regimen. Results: Mean $R_{a}$ values ranged from 0.26 (DuraFlex control) to 1.82 (Valplast adjusted with TC burs). Mean $R_{\max }$ values ranged from 1.88 (Dura Flex control) to 13.76 (Valplast adjusted with TC burs). Two-factor ANOVA revealed that interaction of both factors was significant $(\mathrm{p}<0.05)$ for $R_{a}$ and $R_{\max }$. There was a statistically significant increase in both $R_{a}(\mathrm{p}<0.05)$ and $R_{\max }(\mathrm{p}<0.05)$ for all material types following the gross adjustment. With the exception of DuraFlex, re-polishing of samples that were previously adjusted with TC burs, on the dental lathe produced surfaces that were comparable to control samples. Conclusion: Adjustment of DuraFlex should be kept to a minimum since the adjustment produced the significant surface detriment that could not be corrected with either of the polishing regimens.
\end{abstract}




\section{Keywords}

DuraFlex, DuraCetal, Valplast, Poly Methylmethacrylate, Surface Roughness

\section{Introduction}

Corrective adjustment of denture bases is normally necessary during the delivery procedure. This improves the patient comfort which may affect compliance with denture wear [1]. Following adjustment, rougher surfaces are the result and subsequent finishing and re-polishing of the denture are integral procedures in the delivery protocol to prevent subsequent adhesion of microorganisms [2].

There is the evidence in the literature on the effect of roughened poly methyl methacrylate (PMMA) surfaces on the formation of dental biofilm and subsequent plaque stagnation with sequelae of denture stomatitis, gingival inflammation and the development of root decay on abutment teeth. Authors have demonstrated the adhesion of Candida species and Streptococcus oralis to roughed dental prosthesis fabricated with PMMA [3] [4].

Generally, the process of polishing the adjusted compression molded PMMA denture surfaces, using successively finer grits of pumice and a rag wheel mounted on a lathe has been considered superior to chair side methods for the re-polishing [2]. O'Donnell et al., however, concluded that, where dental clinicians had no access to laboratory facilities, silicone points used in a chair side method, were suitable for smoothening PMMA surfaces [5]. Bollen et al. demonstrated the effect of grit sizes of the polishing armamentarium on the final surface roughness of PMMA [6].

In comparison, the surface roughness of nylon containing polyamide dentures, a thermoplastic denture base, have been investigated both before and after standard lathe polishing and have been found to be significantly rougher than PMMA but fell within an acceptable limit using the in-vivo threshold level of 0.2 $\mu \mathrm{m}$, of average roughness, at which dental plaque begins to accumulate [7].

PMMA resin has been traditionally used for the fabrication of removable dentures due to its ability to evenly distribute masticatory forces to the residual ridges, however, the material demonstrates the poor impact strength and limited use in patients with sensitivity reactions to PMMA [1].

Recently, two injection molded thermoplastic materials: DuraFlex (random copolymer of polypropylene) (Myerson Tooth Company, Chicago, Il, USA) and DuraCetal ((polyoxymethylene) (Myerson Tooth Company, Chicago, Il, USA), based solely on hydrocarbon chemistry, without embedded fibers, have been developed for the use as denture bases and tooth-colored clasps respectively with the aim of improving patient comfort and reducing patient cost. These materials have been purported by the manufacturer to have improved impact strength and have been suggested for use by patients who have sensitivity reactions to PMMA monomer. DuraCetal (polyoxymethylene), mainly used for retentive tooth colored clasps, can be incorporated into significant portions of denture bases.

A search of the literature resulted in no published evidence on the evaluation of surface roughness of DuraCetal and DuraFlex materials as a result of adjust- 
ment and re-polishing or the best armamentarium to be used in the adjustment of these materials.

The aim of this study was to assess the roughness parameters of two newer injection-molded, thermoplastic denture base materials compared with those of a polyamide material and compression molded PMMA before and after adjustment and re-polishing with either a laboratory protocol or a chair side protocol, using contact profilometry and scanning electron microscopy. The null hypothesis stated that there would be no difference in the surface roughness of any of the materials finished with any of the re-polishing regimens.

\section{Materials and Methods}

A waiver of ethical approval was obtained for this study from The University of the West Indies Ethics Committee. A power analysis was completed and determined that 10 samples would be required for each experimental group. Four denture base materials were utilized in this study. A compression molded poly methylmethacrylate (PMMA)-Millennium ${ }^{\circledR}$ (Keystone Industries, Gibbstown, NJ, USA, 08027) and three injection-molded materials-Valplast ${ }^{\star}$ (Valplast International Corp., Long Beach, NY, 11561), DuraFlex ${ }^{\circledast}$ (Myerson Tooth Company Chicago, Il, USA 60640) and DuraCetal ${ }^{\Phi}$ (Myerson Tooth Company, Chicago, Il, USA 60640). Forty specimens $(3 \mathrm{~mm} \times 9 \mathrm{~mm} \times 24 \mathrm{~mm}$ ) each were fabricated, using highly polished stainless steel molds and finished according to individual manufactures' instructions. Valplast, DuraFlex and DuraCetal materials were adjusted with Top Star Brown Rubber Points (Edenta AG, Hauptstrasse 7, Switzerland) to remove flash and then buffed with a dry rag wheel on a lathe (Baldor, Fort Smith, AR, USA, 729$01)$ at $1725 \mathrm{rpm}$. The PMMA samples were polished with decreasing grits of pumice in a wet environment on a lathe following by Tripoli on a rag wheel to achieve a high-shine. All post-fabrication polishing was done by a single operator (AKB).

Forty specimens of each material were randomly assigned to four experimental groups of 10 each.

Group 1 served as the control group with no further adjustment or polishing.

Group 2 specimens were adjusted with standard plain cut tungsten carbide burs (TC) for cutting acrylic (NTI Instruments, IM Camisch 3, 07768, Kahla, Germany) in a unidirectional manner for a total of 30 seconds, using an air driven slow speed straight nose handpiece at 30,000 rpm (Dentsply, York, PA, USA, 17405). One acrylic bur was used for every 10 specimens.

Group 3 specimens was adjusted as described for Group 2 and then re-polished using decreasing grits of silicon carbide impregnated in a synthetic rubber matrix (B-SC) (Maestro Acrylic Polishers, Coarse, Medium and Fine, Henry Schein Inc, Melville, NY, 11747) together with a copious amount of polishing lubricant (Myerson Fine Scratch remover, Myerson Tooth Company, Chicago, Il, USA 60640). Each rubber tip of varying grit was used for a total of 1 minute, for a total polishing time of 3 minutes.

Group 4 specimens were adjusted as described for Group 2 and then re-polished on a dental lathe (Baldor, Fort Smith, AR, 72901) using pumice flour and a rag wheel for 2 minutes at $1725 \mathrm{rpm}$. All samples were adjusted and re-polished 
by one operator $(\mathrm{AKB})$ to reduce the effect on inter-operator variability in terms of the pressure applied during polishing.

\subsubsection{Profilometry}

Following re-polishing the samples were quantitatively assessed with surface profilometry using the Mahr Pocket Surf (Mahr Federal Inc., Provedence, RI, USA, 02905) with a cutoff of $0.8 \mathrm{~mm}$, a transverse length of $5.0 \mathrm{~mm}$ and an evaluation length of $4.0 \mathrm{~mm}$. The probe moved at a transverse speed of 5.08 $\mathrm{mm} /$ second with a force of $15 \mathrm{mN}$ perpendicular to the direction of use of the acrylic bur. Eight parallel tracings, approximately $1.0 \mathrm{~mm}$ apart, were used to assess the surface of the acrylic. For each profile length surface parameters of both $R_{a}$ and $R_{\max }$ were evaluated.

Means and standard deviations were calculated for each group. A two-factor ANOVA was used to determine significant differences in mean surface roughness ( $R_{a}$ and $R_{\max }$ ), with included factors being the material type and the re-polishing regimen. Post-hoc Bonferroni was used for pairwise comparison of the experimental groups. All statistical analysis was carried out with SPSS Version 21 (IBM Corporation, Armonk, NY, USA, 10022).

\subsubsection{Scanning Electron Microscopy (SEM) Analysis}

Two specimens from each group were randomly selected for qualitative assessment via SEM. A $5 \mathrm{~mm} \times 5 \mathrm{~mm}$ section was carefully removed from the corner of each specimen, ultrasonically cleaned in distilled water for 5 mins (Branson 2210, Branson Ultrasonics Corporation, Danbury, Connecticut) to remove any debris and sputter coated with gold (DentonVacuum LLC, Moorestown, NJ, USA) to facilitate SEM analysis. Photomicrographs were taken at magnifications between $\times 300$ and $\times 3500$. Depending on the size and pattern of surface alterations the micrographs were classified as (1) smooth/acceptable surface (2) minor surface detriment (3) major surface detriment.

\section{Results}

\section{1. $R_{a}$ Measurements}

The means and standard deviations for $R_{a}$ readings can be seen in Table 1. Mean $R_{a}$ readings ranged from 0.26 (DuraFlex control) to 1.82 (Valplast adjusted with acrylic bur). Statistical tests revealed that interaction of both factors were significant $(\mathrm{F}=68.95, \mathrm{p}<0.05)$ for $R_{a}$. There were significant increases in $R_{a}$ for all the materials when adjusted with TC $(\mathrm{p}<0.001)$. Control samples demonstrated significant differences between the average roughness of PMMA and DuraFlex ( $\mathrm{p}<$ $0.05)$ and between PMMA and DuraCetal $(\mathrm{p}<0.05)$ while there was no difference in the roughness of DuraCetal and DuraFlex $(\mathrm{p}=1.00)$. Furthermore, the $R_{a}$ of Valplast was significantly higher than either DuraCetal, DuraFlex and PMMA control materials ( $\mathrm{p}<0.001$, for all control pairwise comparisons).

TC adjusted PMMA samples when re-polishing with either a lab based regimen $(\mathrm{p}<0.001)$ or chair side B-SC $(\mathrm{p}<0.001)$ resulted in significantly reduced roughness, compared even to the control non-adjusted specimens. 
Table 1. Mean ( $\pm \mathrm{SD})$ Roughness values $\left(R_{\mathrm{a}}\right)$ for tested materials $(\mu \mathrm{m})$.

\begin{tabular}{ccccc}
\hline \multicolumn{5}{c}{ Polishing Regimen } \\
\hline & (Group 1) & (Group 2) & (Group 3) & (Group 4) \\
\hline Material Type & Control & TC-Adjustment & B-SC Polishing & Pumice and Rag Wheel \\
\hline PMMA & $0.96(0.62)$ & $1.47(0.44)$ & $0.63(0.52)$ & $0.42(0.37)$ \\
Valplast & $1.30(0.54)$ & $1.82(0.32)$ & $1.19(0.22)$ & $0.79(0.30)$ \\
DuraFlex & $0.26(0.14)$ & $1.08(0.42)$ & $0.52(0.24)$ & $0.52(0.23)$ \\
DuraCetal & $0.30(0.11)$ & $1.20(0.35)$ & $0.95(0.49)$ & $0.50(0.17)$ \\
\hline
\end{tabular}

Valplast control samples were significantly rougher than all the other tested control materials, with TC adjustment causing significant detriment to the surface in terms of roughness $(\mathrm{p}<0.001)$. These surfaces, however, were subsequently significantly improved when subjected to lathe polishing or chair-side B-SC polishing with lathe polishing improving the surface quality beyond that of control samples $(\mathrm{p}<0.001)$. The surface quality of adjusted Valplast samples polished with B-SC polishers was comparable to control samples $(\mathrm{p}=0.91)$.

Control DuraFlex material was as rough as the control DuraCetal material ( $\mathrm{p}$ $=1.00$ ), with the lowest observed $R_{a}$ values. TC adjusted DuraFlex material was significantly improved with either lathe $(\mathrm{p}<0.001)$ or B-SC re-polishing $(\mathrm{p}<$ 0.001 ) however, these surfaces were still both rougher compared to control samples $(\mathrm{p}=0.002)$.

TC adjusted DuraCetal showed a significant reduction in $R_{a}$ when re-polished on the lathe $(\mathrm{p}<0.001)$ or with B-SC polishers $(\mathrm{p}=0.002)$ Lathe re-polishing produced surfaces that were comparable to control samples $(p=0.67)$.

\section{2. $\boldsymbol{R}_{\max }$ Measurements}

The means and standard deviations for $R_{\max }$ can be seen in Table 2. Mean $R_{\max }$ readings ranged from $1.88 \mu \mathrm{m}$ (DuraFlex Control) to $13.76 \mu \mathrm{m}$ (Valplast adjusted with acrylic burs). The two factor analysis of variance revealed that interaction of the two factors was significant $(F=87.84$ and $p<0.05)$ for both of the tested parameters. There was a significant increase in $R_{\max }$ values for all the tested materials as a result of adjustment with TC burs. Conversely, for all materials adjusted and subsequently re-polished there were significant reductions in $R_{\max }$ values compared to TC adjusted samples only.

Post hoc analysis demonstrated that PMMA samples adjusted with TC burs and re-polished with B-SC tips ( $\mathrm{p}<0.001)$ or on the lathe $(\mathrm{p}<0.0001)$ significantly improved the $R_{\max }$ values compared to controls.

TC adjusted Valplast samples subsequently re-polished with B-SC tips ( $\mathrm{p}<$ $0.001)$ or on the lathe $(\mathrm{p}<0.001)$ were also significantly improved compared to control samples. Samples re-polished on the lathe produced smoother surfaces compared to B-SC polishing ( $\mathrm{p}<0.001)$.

$R_{\max }$ values significantly improved for lathe $(\mathrm{p}<0.001)$ or B-SC polishing ( $\mathrm{p}<$ 0.001 ) of DuraFlex adjusted samples, with both re-polishing regimens showing 
Table 2. Mean $( \pm \mathrm{SD})$ Maximum Roughness values $\left(R_{\max }\right)$ for tested materials $(\mu \mathrm{m})$.

\begin{tabular}{ccccc}
\hline \multicolumn{5}{c}{ Polishing Regimen } \\
\hline & (Group 1) & (Group 2) & (Group 3) & (Group 4) \\
\hline Material Type & Control & TC-Adjustment & B-SC Polishing & Pumice and Rag Wheel \\
\hline PMMA & $6.69(3.97)$ & $9.64(1.96)$ & $2.89(1.14)$ & $2.57(2.02)$ \\
Valplast & $8.44(3.82)$ & $13.76(2.55)$ & $7.50(1.37)$ & $5.09(1.63)$ \\
DuraFlex & $1.88(1.19)$ & $10.57(2.07)$ & $3.01(0.98)$ & $3.56(1.34)$ \\
DuraCetal & $2.47(1.21)$ & $9.90(2.30)$ & $5.06(2.14)$ & $3.24(0.93)$ \\
\hline
\end{tabular}

similar results (lathe versus B-SC polishing, $\mathrm{p}=1.00$ ). Unlike analogous PMMA and Valplast, samples, re-polished surfaces of adjusted DuraFlex were significantly rougher compared to control ( $\mathrm{p}<0.001$ for both regimens).

$R_{\max }$ values for TC adjusted DuraCetal were significantly reduced with either lathe ( $<<0.001)$ or B-SC tip re-polishing ( $<0.001)$, with lathe polishing producing a statistically improved surface result (lathe versus B-SC tip, $\mathrm{p}<0.001$ ). Lathe re-polished DuraCetal was as smooth as control samples $(\mathrm{p}=1.00)$

\subsection{SEM Analysis}

Qualitative assessment of control surfaces (Figures 1(a)-1(d)) correlated well with surface roughness measurements. Examination revealed Valplast to be the material demonstrating the most surface detriment, while DuraFlex and DuraCetal samples were relatively smooth.

Group 2 samples, adjusted with TC burs demonstrated major surface detriment; again with Valplast samples being the worse (Figures 2(a)-2(d)). High magnification SEM analysis revealed prominent loosening of nylon fibers on the surface of the material when adjustment took place (Figure 2(a)). DuraFlex and DuraCetal showed evidence of delamination of surface material as a result of adjustment with TC burs (Figure 2(c), Figure 2(d)).

Group 3 samples showed an improvement in surface quality compared to their Group 2 counterparts (Figures 3(a)-3(d)). Micrographs of PMMA clearly showed large surface defects related to the manipulation and processing of the material (Figure 3(a-ii)). Exposed nylon fibers were still evident on the Valplast samples despite showing an improvement in overall surface quality (Figure 3(b)). DuraFlex samples showed evidence of embedded particles of silicone carbide on examined surfaces.

Group 4 samples also showed an improvement in surface quality compared to analogous Group 2 samples however they were not comparable to control (Group 1) samples (Figures 4(a)-4(d)). PMMA, Valplast and DuraCetal samples all showed minor surface alteration as a result of re-polishing on the dental lathe (Figure 4(a), Figure 4(b), Figure 4(d)). DuraFlex re-polished on the dental lathe showed major surface detriment (Figure 4(c)). 


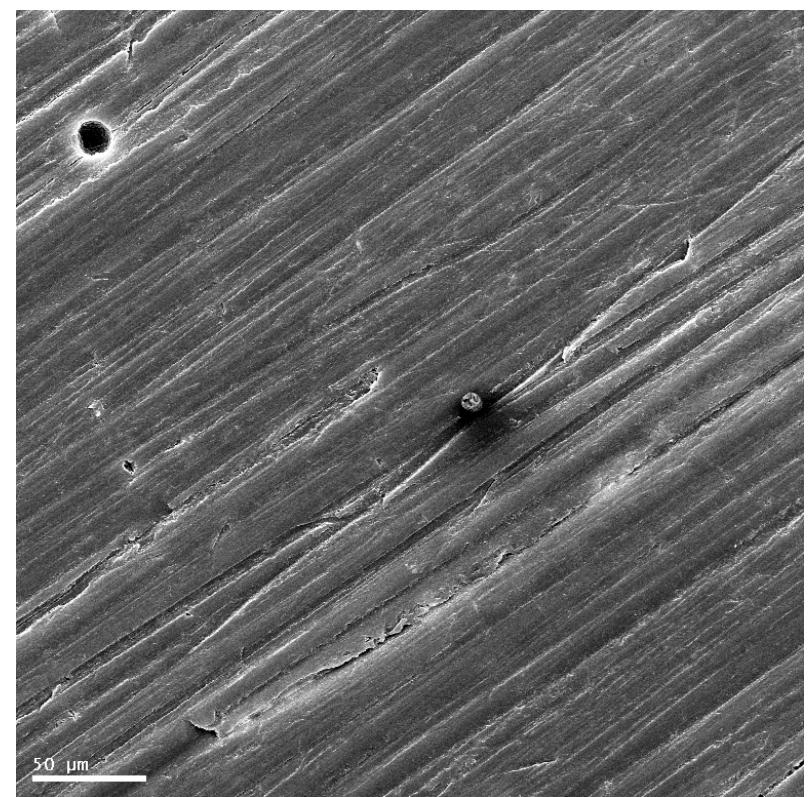

(a)

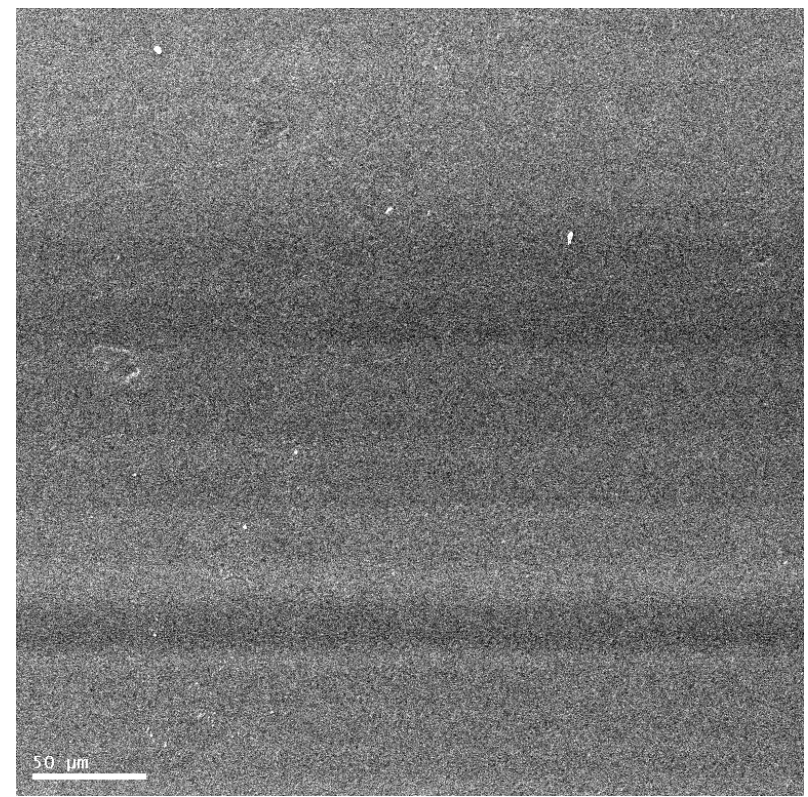

(c)

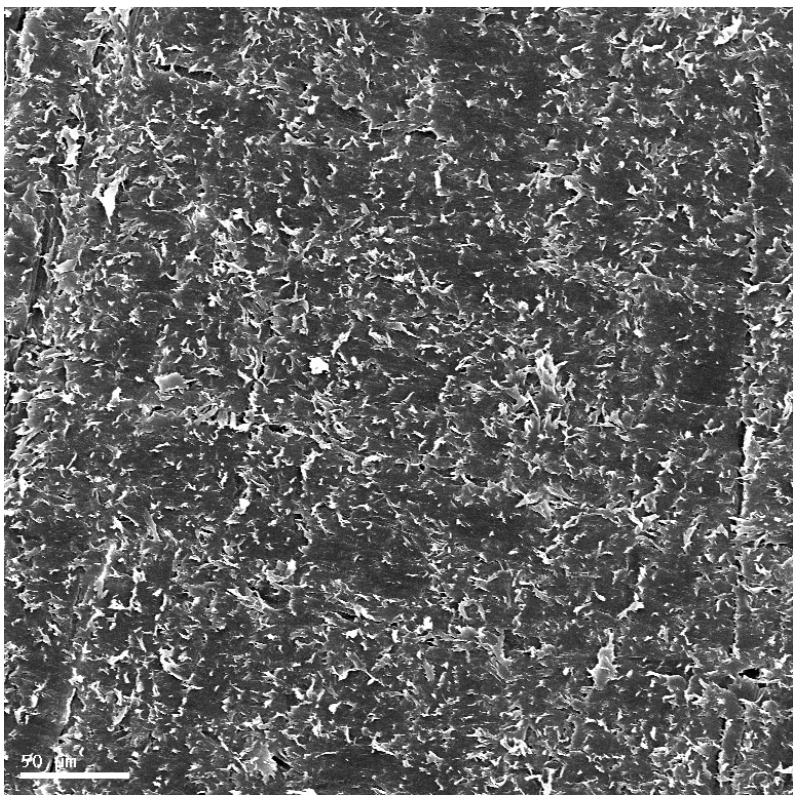

(b)

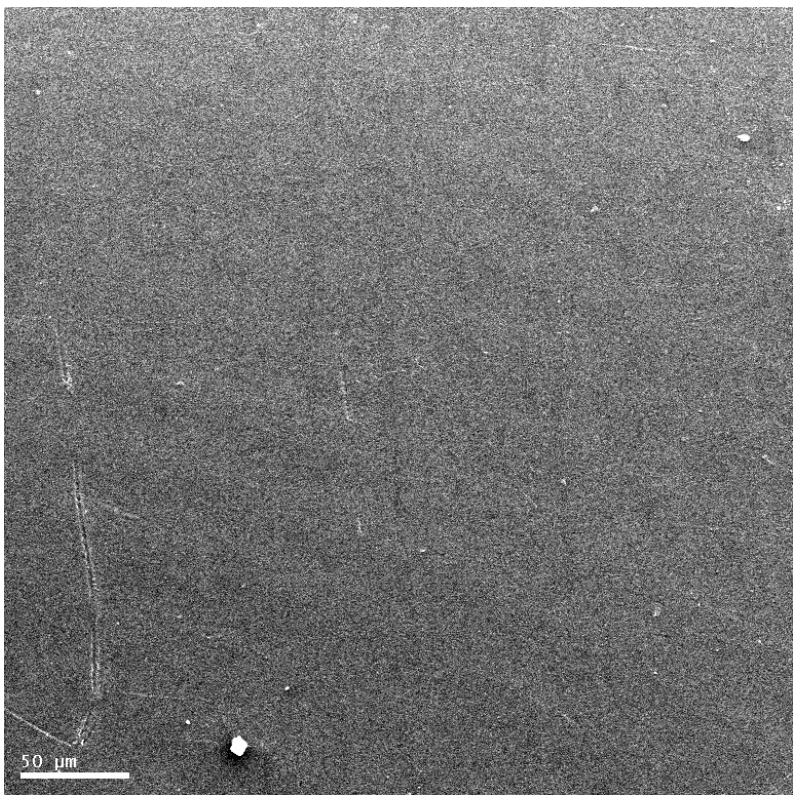

(d)

Figure 1. (a) Scratches associated with gross adjustment with tungsten carbide cuttings burs. $(\times 300)$ Large voids are seen in upper right hand corner as a result of processing error. (b) Control Valplast surface showing major surface detriment $(\times 300)$. $(c)$ Smooth, homogenous surface of control DuraFlex surface $(\times 350)$. (d) Smooth, homogenous surface of control DuraCetal surface $(\times 300)$.

\section{Discussion}

Contact profilometric tracing involves the movement of a stylus across the surface of the tested material over a specific distance called Profile length. This movement results in vertical displacement of the stylus following the peaks and valleys of the material surface characterizing it at the micro-meter scale. The values obtained include the Average Roughness, $R_{a}$ which is the mean of several vertical displacements of the stylus and the Maximum Roughness, $R_{\max }$ which is 


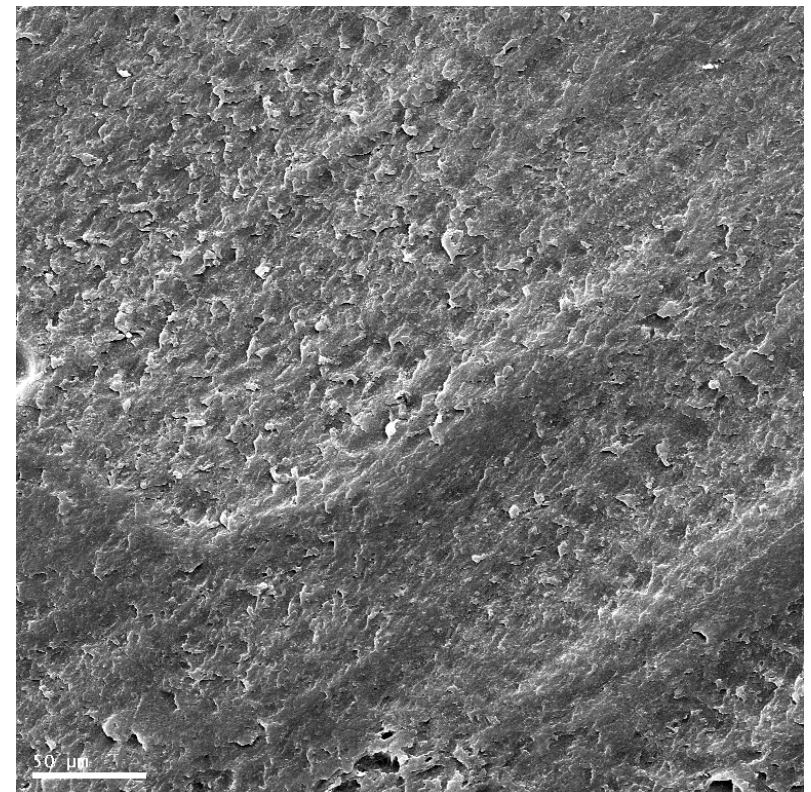

(a)

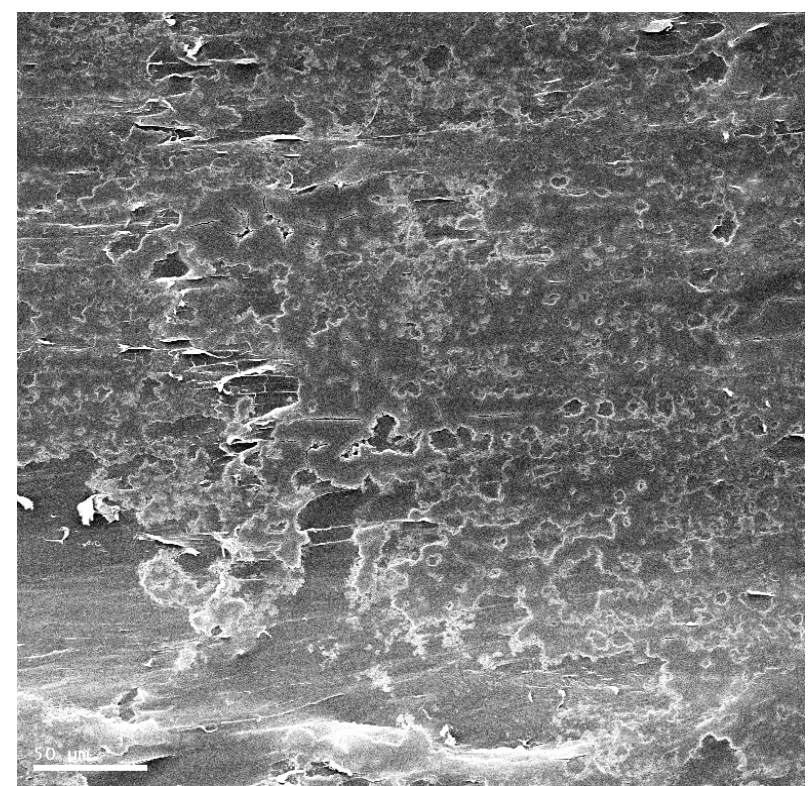

(c)

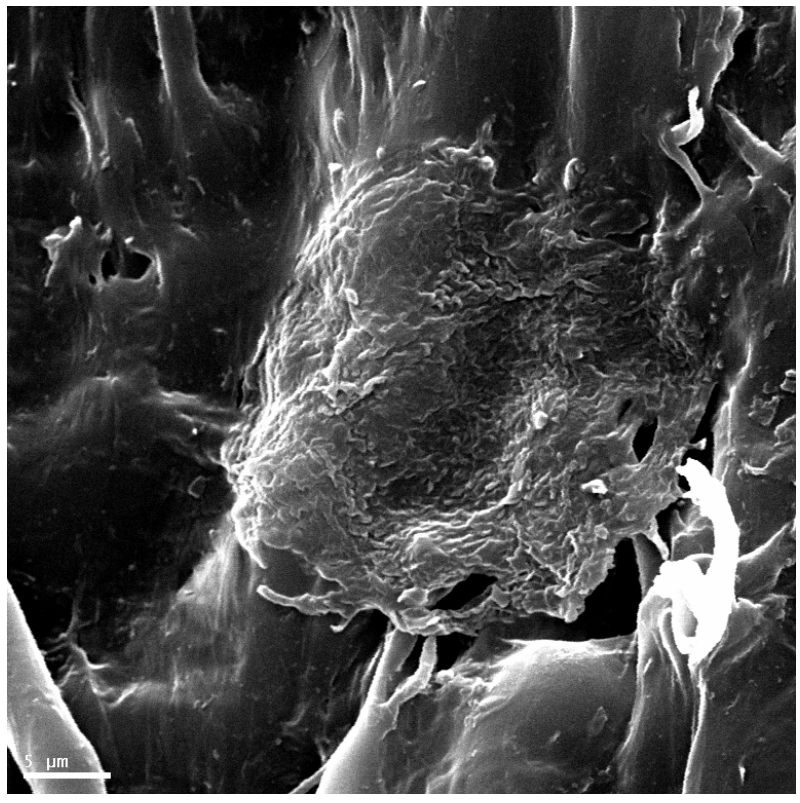

(b)

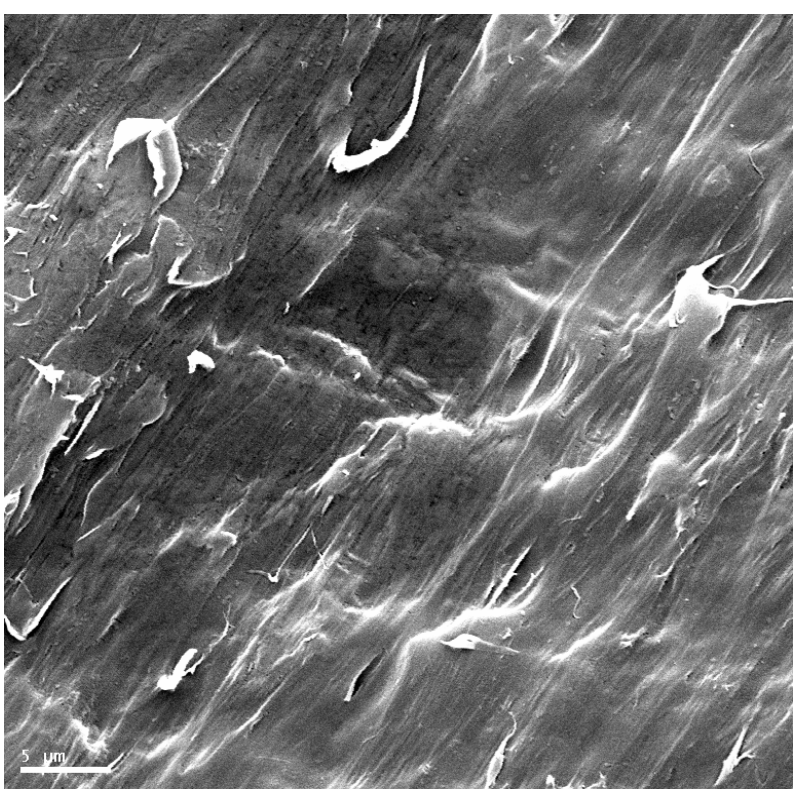

(d)

Figure 2. (a) PMMA surface adjusted with TC burs, showing wide scratches as a result of instrumentation. ( $\times 350)$; (b) Valplast surface adjusted with TC burs, with evidence of loosened nylon fibers. $(\times 2700)$; (c) DuraFlex surface adjusted with TC, with evidence of delamination of the surface. $(\times 300)$; (d) DuraCetal surface adjusted with TC, with evidence of marked delamination of the surface. $(\times 2700)$.

the maximum displacement encountered and which is also known as Extreme Value Descriptor [8].

Various authors in the dental literature have advocated the use of more than one roughness parameter in determining surface characterization when more than one finishing/polishing instrument is used [9] [10]. The present study, however, demonstrated that the $R_{a}$ readings closely mirrored $R_{\max }$ readings and no real benefit was noted from using two roughness parameters despite the use of TC burs followed by the re-polishing protocols. 


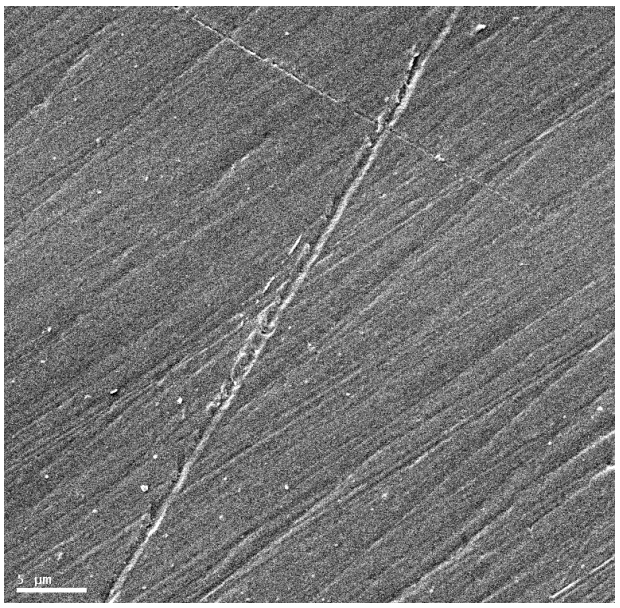

(i)

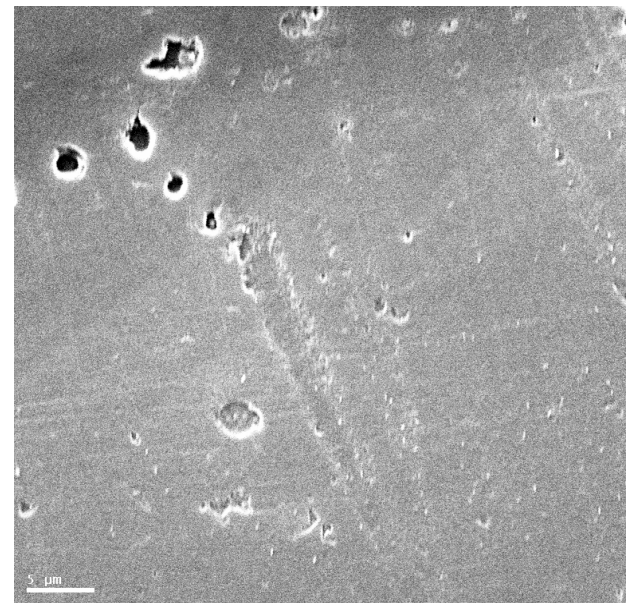

(ii)

(a)

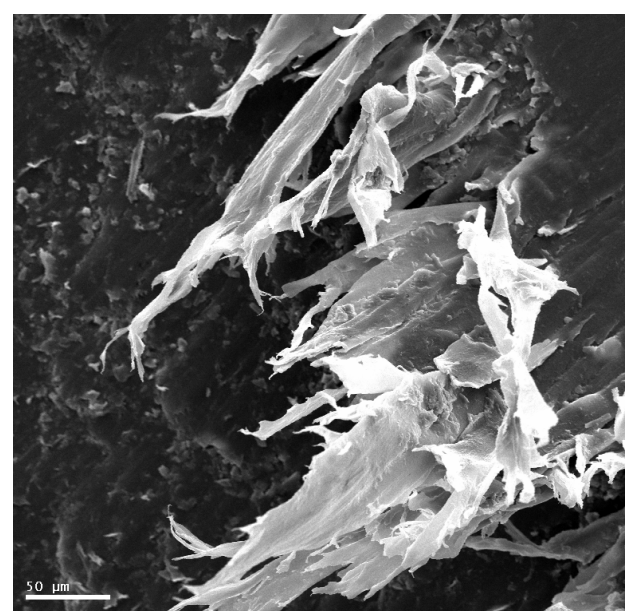

(b)

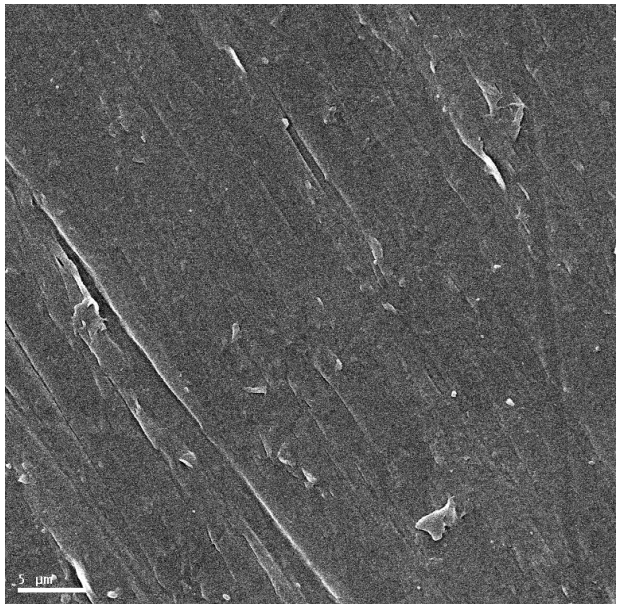

(c)

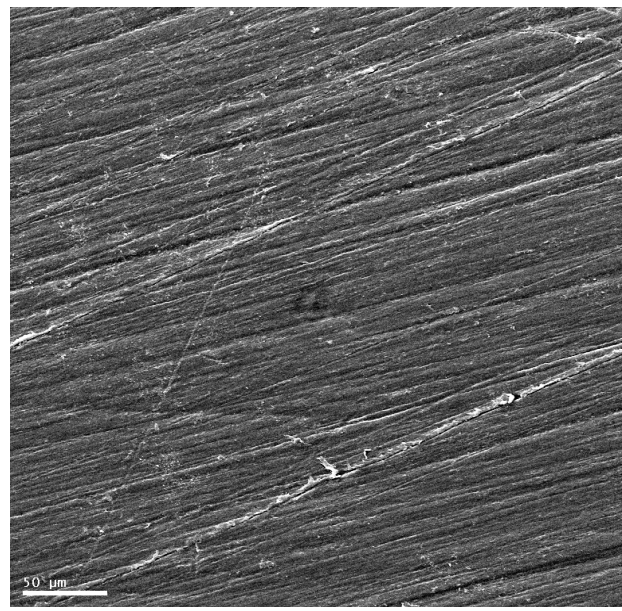

(d)

Figure 3. (a-i) TC adjusted PMMA surface re-polished with B-SC polishers. The surface quality is improved compared to control. $(\times 2700)$; (a-ii) PMMA surface repolished with B-SC polishers. Note the voids as a result of errors compression molded processing technique. $(\times 2700)$; (b) Loose nylon fibers present despite re-polishing Valplast with B-SC polishers. $(\times 300)$; (c) DuraFlex re-polished with B-SC polishers showing minor surface detriment compared to control samples. $(\times 300)$; (d) DuraCetal re-polished with B-SC polishers showing major surface detriment $(\times 300)$. 


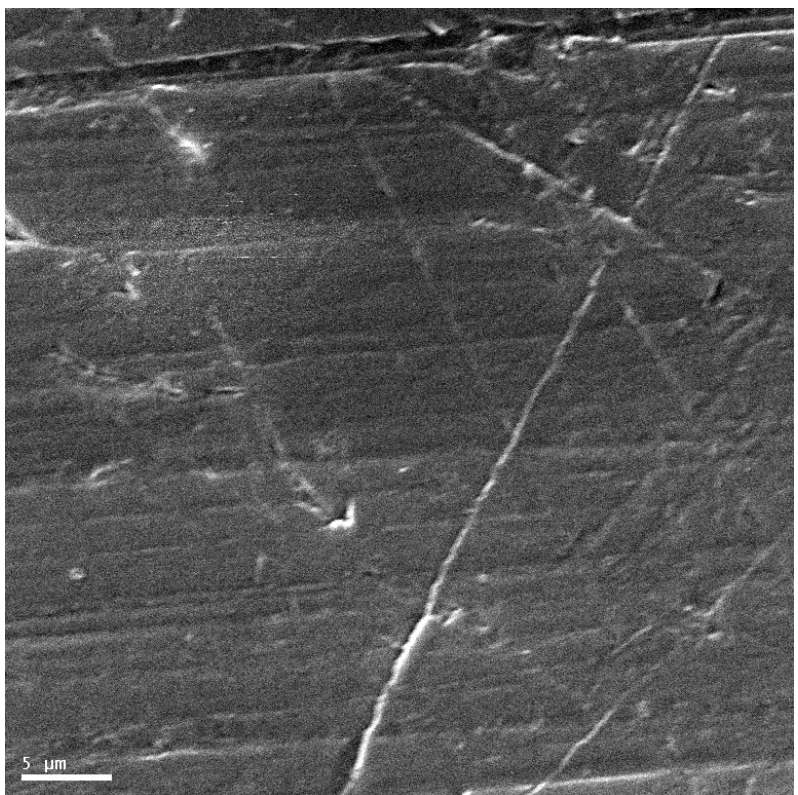

(a)

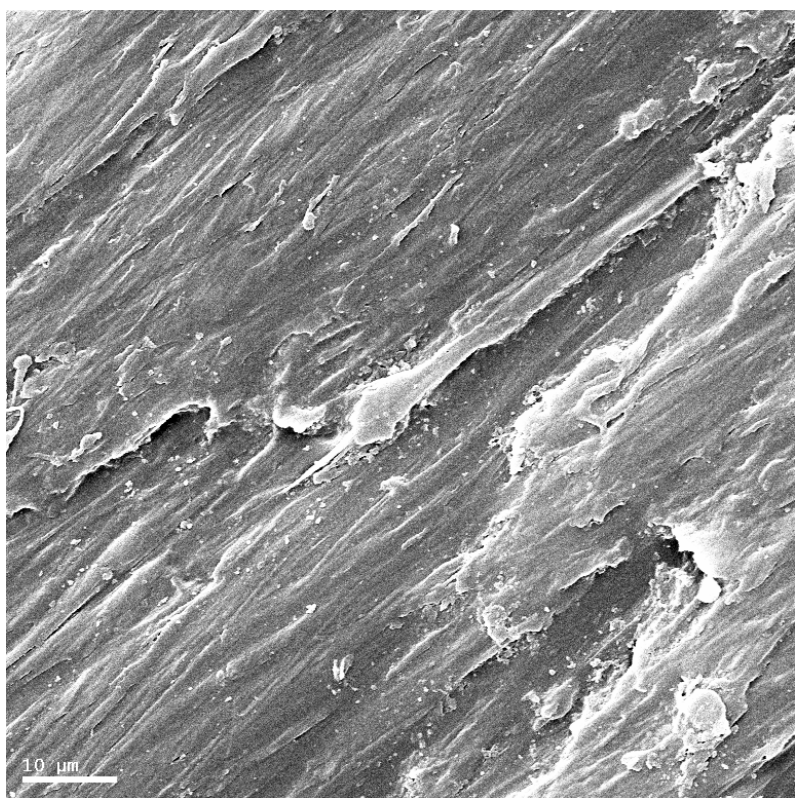

(c)

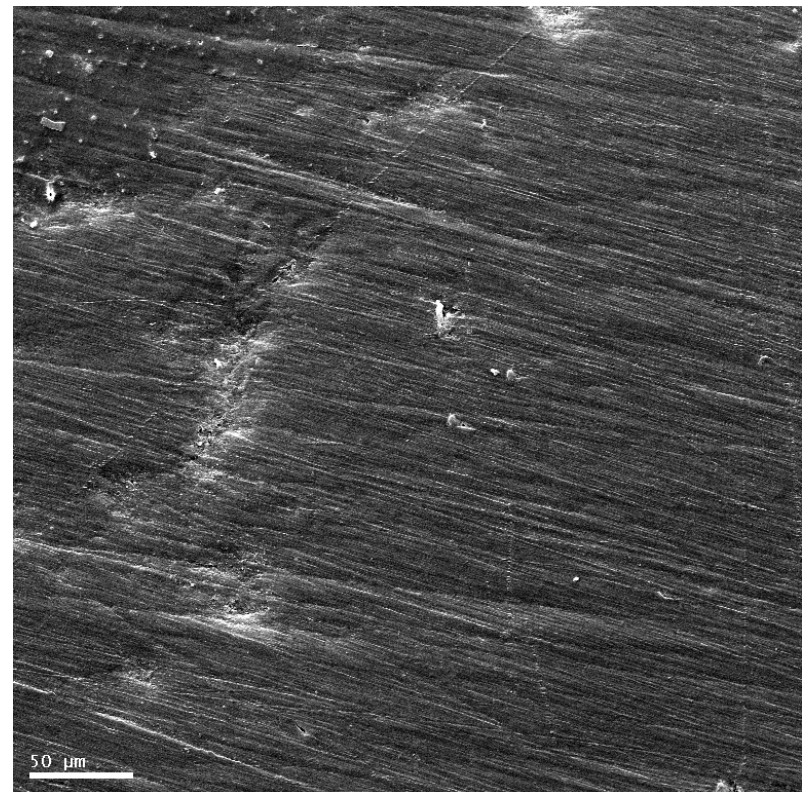

(b)

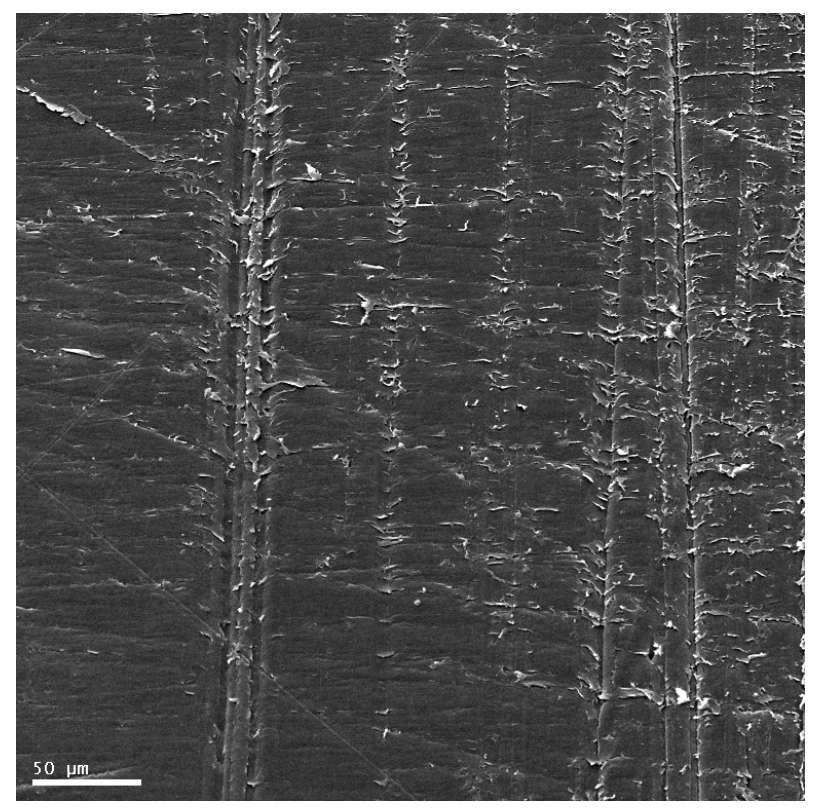

(d)

Figure 4. (a) PMMA re-polished on the lathe. Compared to the analogous control photomicrograph the surface shows minor surface detriment. $(\times 2700)$; (b) Valplast re-polished on the lathe. Compared to the analogous Group 2 photomicrograph the surface shows minor surface detriment. $(\times 300)$; (c) DuraFlex re-polished on the lathe. The surface shows structural defects in addition to scratches associated with TC bur adjustment. $(\times 1400)$; (d) DuraCetal re-polished on the lathe. Compared to the analogous control photomicrograph, the surfaces show the minor surface detriment. $(\times 300)$.

With the exception of Valplast, this study demonstrated the superiority of injection molded techniques versus compression molded techniques with respect to fabricating denture bases as evidenced by the variations in surface quality of control PMMA compared with either the DuraCetal or DuraFlex material. Small discrepancies in the manipulation of monomer liquid with polymer powder can result in sub-optimum surface properties, this was clearly evidenced in Figure 
3(a-ii). Conversely, uniform melting and injection molding of both DuraFlex and DuraCetal, during processing, resulted in more homogenous control surfaces.

Surface roughness of materials following instrumentation contributes significantly to overall surface quality. Variations in the microstructure of the material together with mechanical properties such as microhardness and modulus of elasticity may also affect the surface quality. The photomicrograph of control Valplast with exposed nylon fibers (Figure 1(b)) clearly demonstrates how variations in microstructure can affect surface quality.

Generally, with the exception of DuraFlex, samples, lathe polishing using pumice and rag wheel produced superior results in terms of roughness measurements compared to B-SC polishing. This could be to the greater depth of action of the unbonded pumice particles in removing surface scratches left behind by the use of TC burs. The B-SC instrument, due to the fact that at least half of the abrasive particle is embedded in the synthetic rubber carrier, limits the penetration of the abrasive particle into the polymer substrate thereby not efficiently removing surface defects caused by gross adjustment with TC burs [11].

Adjustment and re-polishing of the PMMA significantly improved surface quality, with lathe polishing producing better results. This finding concurs with that of Berger et al. who concluded that conventional lab polishing produced superior results regardless of the type of PMMA [2].

The finding with respect to the control polyamide Valplast material concurs with that of Abuzar et al., who demonstrated rougher surfaces compared to PMMA [7]. In this present study there was a reduction by just over half the $R_{a}$ values of Valplast after polishing with pumice and rag-wheel on the lathe which is well over the in-vivo threshold value of $0.2 \mu \mathrm{m}$ discussed in the literature, which facilitates microbial adhesion on acrylic materials [12] [13]. This is in contrast to Abuzar et al., where a 7 fold decrease in $R_{a}$ values as a result of using pumice on the lathe, was noted [7]. This could be explained by differences in methodology in terms of the adjustment and finishing materials used, and operator variability in terms of pressure applied during polishing.

Polyamide dentures, such as Valplast have a low melting point and adjusting and polishing in a dry environment with the B-SC tips could potentially cause overheating of the surface which may cause loosening and exposure of the nylon fibers, which translates into increased surface roughness. In contrast the pumice slurry was used in a typical wet environment which could have lessened the effect of surface heating and subsequent surface detriment. It is noteworthy that even though TC burs were used in the methodology; which are contra-indicated for adjusting polyamide dentures, subsequent lab polishing was able to reduce average roughness within the range of $0.7-1.0 \mu \mathrm{m}$. quoted by various authors, for bacterial adhesion [14] [15], DuraFlex and DuraCetal produced very smooth surfaces prior to adjustment and re-polishing with high surface roughness values once any adjustment and re-polishing was completed. The authors recommend careful impression technique, waxing and fabrication of dentures in an attempt to minimize the need for gross adjustment. 
Generally, though the surface of polymeric materials are dependent on temperature due to their poor thermal conductivity and the friction of finishing and polishing temperatures [16]. Both DuraFlex (random copolymer of polypropylene) and DuraCetal (polyoxymethylene) are considered thermoplastic, amorphous polymers with random orientation of chain structure. Such materials are prone to thermal energy with stretching and bending of chains which can lead to further degradation of the surface unrelated to the scratches associated with the abrasive materials of either the bonded (silicone tips) or un-bonded (pumice) abrasives. The visualization of the phenomenon of delamination, observed in Figure 2(c) and Figure 2(d), in addition to roughened surfaces could be attributed to such increases in temperature.

Lubricants are useful in polishing procedures to reduce friction and dissipate heat [17]. In this current study the authors have postulated that the use of the polishing lubricant was ineffective in dissipating heat produced during the re-polishing procedures during the use of B-SC. Generally water may be a useful lubricant alternative with any of the tested materials. Specifically, with respect to DuraFlex, the use of the hydrocarbon oils such as squalene or isododecane, with similar polarity, may be useful as polishing lubricants.

Further areas of research should also include an assessment of the microhardness of the processed materials, since this important property influences the efficiency of removal of material from surfaces being polished and could inform on the best type of cutting instruments that should be used in the gross adjustment of such materials.

Another area of research should focus on the critical roughness values required for the initiation of bacterial and fungal adhesion on these newer injection molded materials since there is considerable variation in the literature with respect to the cited roughness values for adhesion of various microorganisms [12] [13] [14] [15].

\section{Conclusions}

Within the limitations of this study, the following conclusions may be drawn:

1) All denture base materials when adjusted with TC burs showed an increase in $R_{a}$ and $R_{\max }$.

2) Valplast showed the greatest roughness values in terms of both $R_{a}$ and $R_{\max }$ values before and after the adjustment and re-polishing.

3) Adjustment of DuraFlex materials should be kept to a minimum to maintain the surface quality achieved following injection molding since all re-polishing regimes produced the significant surface detriment.

4) With the exception of DuraFlex, material lathe polishing is superior to chair side polishing with B-SC.

\section{Conflict of Interest}

The authors have no financial or proprietary interest in any of the products, techniques and materials discussed in this research report. 


\section{Acknowledgements}

This research was made possible with a research grant from the University of the West Indies, Campus Research and Publication Fund (Grant No. CRP.3.JUN15.6).

The authors would like to thank Myerson Tooth Company for the generous donation of materials used in this research project.

The authors would also like to thank Dr. Virendra Dhuru for his review and editing of the manuscript.

\section{References}

[1] Kuhar, M. and Funduk, N. (2005) Effects of Polishing Techniques on the Surface Roughness of Acrylic Denture Base Resins. The Journal of Prosthetic Dentistry, 9 , 76-85. https://doi.org/10.1016/j.prosdent.2004.10.002

[2] Berger, J.C., Driscoll, C.F., Romberg, E., Luo, Q. and Thompson, G. (2006) Surface Roughness of Denture Base Acrylic Resins after Processing and after Polishing. Journal of Prosthodontics, 15, 180-186. https://doi.org/10.1111/j.1532-849X.2006.00098.x

[3] Verran, J. and Maryan, C.J. (1997) Retention of Candida albicans on Acrylic Resin and Silicone of Different Surface Topography. Journal of Prosthetic Dentistry, 77, 535-539. https://doi.org/10.1016/S0022-3913(97)70148-3

[4] Radford, D.R., Sweet, S.P., Challacombe, S.J. and Walter, J.D. (1998) Adherence of Candida albicans to Denture-Base Materials with Different Surface Finishes. Journal of Dentistry, 26, 577-583. https://doi.org/10.1016/S0300-5712(97)00034-1

[5] O’Donnell, E.F., Radford, D.R., Sinclair, G.F. and Clark, R.K.F. (2003) Chairside Polishing of Heat-Cured Acrylic Resin: An SEM and EDA Study. The Journal of Prosthetic Dentistry, 90, 585. https://doi.org/10.1016/j.prosdent.2003.08.014

[6] Bollen, C.M., Lambrechts, P. and Quirynen, M. (1997) Comparison of Surface Roughness of Oral Hard Materials to the Threshold Surface Roughness for Bacterial Plaque Retention: A Review of the Literature. Dental Materials, 13, 258-269. https://doi.org/10.1016/S0109-5641(97)80038-3

[7] Abuzar, M.A., Bellur, S., Duong, N., Kim, B.B., Lu, P., Palfreyman, N., Surendran, D. and Tran, V.T. (2010) Evaluating the Surface Roughness of a Polyamide Denture Base Material in Comparison with Poly(Methyl Methacrylate). Journal of Oral Science, 52, 577-581. https://doi.org/10.2334/josnusd.52.577

[8] Marchan, S.M., White, D., Marchan, Q.M., Dhuru, V. and Smith, W.A. (2013) Surface Properties of Dental Nanocomposites after Finishing with Rigid Rotary Instruments. Operative Dentistry, 38, 519-527. https://doi.org/10.2341/12-214-L

[9] Baseran, M. (2004) Surface Roughness of Nanofill and Nanohybrid Composite and Ormocer Based Tooth Colored Restorative Materials after Several Finishing and Polishing Procedures. Journal of Biomaterials Applications, 19, 121-134. https://doi.org/10.1177/0885328204044011

[10] Jung, M., Sehr, K. and Klimek, J. (2007) Surface Texture of Four Nanofilled and One Hybrid Composite after Finishing. Operative Dentistry, 32, 45-52. https://doi.org/10.2341/06-9

[11] Darvell, B.W. (2009) Cutting, Abrasion and Polishing. In: Materials Science for Dentistry, 9th Edition, Woodhead Publishing in Ltd., Cambridge, 453.

[12] Quriynen, M., Marechal, M., Busscher, H.J., Weerkamn, A.H., Darius, P.L. and van Steeberghe, D. (1990) The Influence of Surface Free Energy and Surface Roughness on Early Plaque Formation. An in Vivo Study in Man. Journal of Clinical Periodon- 
tology, 17, 138-144. https://doi.org/10.1111/j.1600-051X.1990.tb01077.x

[13] Radford, D.R., Challacombe, S.J. and Walter, J.D. (1999) Denture Plaque and Adherence of Candida albicans to Denture Base Materials in Vivo and in Vitro. Critical Reviews in Oral Biology \& Medicine, 10, 99-116. https://doi.org/10.1177/10454411990100010501

[14] Shintani, H., Satou, N., Hayashihara, A. and Inoue, T. (1985) Effects of Various Finishing Methods of Staining and Accumulation of Streptococcus mutans HS-6 on Composite Resin. Dental Materials, 1, 225-227. https://doi.org/10.1016/S0109-5641(85)80046-4

[15] Weitman, R.T. and Eames, W.B. (1975) Plaque Accumulation on Composite Surfaces after Various Finishing Procedures. The Journal of the American Dental Association, 91, 101-106. https://doi.org/10.14219/jada.archive.1975.0294

[16] Darvell, B.W. (2009) Cutting, Abrasion and Polishing. In: Materials Science for Dentistry, 9th Edition, Woodhead Publishing in Ltd., Cambridge, 455.

[17] Al-Kheraif, A.A. (2014) The Effect of Mechanical and Chemical Polishing Techniques on the Surface Roughness of Heat-Polymerized and Visible Light-Polymerized Acrylic Denture Base Resins. The Saudi Dental Journal, 26, 56-62.

https://doi.org/10.1016/j.sdentj.2013.12.007

Submit or recommend next manuscript to SCIRP and we will provide best service for you:

Accepting pre-submission inquiries through Email, Facebook, LinkedIn, Twitter, etc. A wide selection of journals (inclusive of 9 subjects, more than 200 journals)

Providing 24-hour high-quality service

User-friendly online submission system

Fair and swift peer-review system

Efficient typesetting and proofreading procedure

Display of the result of downloads and visits, as well as the number of cited articles

Maximum dissemination of your research work

Submit your manuscript at: http://papersubmission.scirp.org/

Or contact ojst@scirp.org 